Physiotherapy

\section{Supervised physiotherapy after arthroscopic partial meniscectomy: is it effective?}

\section{P C Goodwin, M C Morrissey}

Much more evidence is required to judge whether supervised physiotherapy is effective after arthroscopic partial meniscectomy

O ur recently published finding that a supervised physiotherapy programme plus written and verbal advice after arthroscopic partial meniscectomy surgery is no more effective than written and verbal advice alone $^{1}$ has caused much interest and debate. $^{2}$ More recently, public debate has arisen from the study by Frost et al, ${ }^{3}$ reporting in the same vein that routine physiotherapy seems to be no more effective than one session of assessment and advice for low back pain. These results should be applied to clinical practice with caution.

In our work, we evaluated the effectiveness of one method of supervised physiotherapy among an infinite number of programmes that can be used. We found this particular method to not be any more effective than written and verbal advice alone. What we did not find was that supervised physiotherapy is ineffective in this patient population-rather, we found that one method of supervised physiotherapy is ineffective when applied to one group of patients. So, considering our findings and the work of others in this area, ${ }^{4-11}$ what is the take home message in terms of offering supervised physiotherapy after arthroscopic partial meniscectomy surgery? We strongly believe that the most important next step is for clinicians to compare the results they obtain in the care they offer these patients with the results in our study. If clinicians are consistently finding that they are no more effective with their care than that which was found in our study, they should either discontinue this care or modify and re-evaluate. On the other hand, if clinicians are consistently finding that they are more effective with their care than that which was found in our study, they should fully evaluate this with a randomised controlled trial and report their findings with extra care to describe the treatment programme given.

In reviews of studies that have included a routine supervised treatment programme after arthroscopic partial meniscectomy, ${ }^{12-14}$ a common criticism is that supervised treatment protocols are not sufficiently described, and where they are described, they are so different that they cannot be compared across studies. Where a supervised treatment protocol is included, the alternative, usually written and verbal advice is also insufficiently described. Poorly described protocols do not benefit our understanding of treatments, nor do they assist clinicians in the evaluation of their own practice.

We believe it is still early days in evaluating the effectiveness of supervised physiotherapy after arthroscopic partial meniscectomy. We do not believe that a definitive study in this area exists nor do we expect that one is forthcoming. We believe that it is naïve to expect a definitive study in the near future. Instead, what is needed and what we expect is a series of studies investigating different treatment programmes applied in this patient population. Only after an exhaustive series of studies have been completed can one judge whether supervised physiotherapy is effective after arthroscopic partial meniscectomy.

\section{Br J Sports Med 2005;39:692.}

doi: $10.1136 /$ bjsm.2004.016790
Authors' affiliations

P C Goodwin, Manchester Metropolitan University, Hathersage Road, Manchester MI3 OJA, UK

M C Morrissey, King's College London, Shepherds House, Guys Campus, London SEI 1UL, UK

Correspondence to: Dr Morrissey; matt.morrissey@kcl.ac.uk

Accepted 29 March 2005

Competing interests: none declared

\section{REFERENCES}

1 Goodwin PC, Morrissey MC, Omar RZ, et al. Effectiveness of supervised physical therapy in the early period after arthroscopic partial meniscectomy. Physical Therapy 2003;83:520-35.

2 Pua Y, Goodwin PC, Morrissey MC, et al. An alternative explanation... Goodwin et al titled "Effectiveness of supervised physical therapy in the early period after arthroscopic partial meniscectomy". Physical Therapy 2003;83:947-8

3 Frost H, Lamb SE, Doll HA, et al. Randomised controlled trial of physiotherapy compared with advice for low back pain. BMJ 2004;329:708.

4 Jensen JE, Conn RR, Hazelrigg G, et al. The use of transcutaneous neural stimulation and isokinetic testing in arthroscopic knee surgery. Am J Sports Med 1985;13:27-33

5 Williams RA, Morrissey MC, Brewster CE. The effects of electrical stimulation on quadriceps strength and thigh circumference in meniscal patients. J Orthop Sports Phys Ther 1986;8:143-6

6 Jokl P, Stull PA, Lynch K, et al. Independent home versus supervised rehabilitation following arthroscopic knee surgery: a prospective randomised trial. Arthroscopy 1989:5:298-305.

7 St Pierre DMM, Laforest S, Paradis S, et al. Isokinetic rehabilitation after arthroscopic meniscectomy. Eur J Appl Physiol 1992:64:437-43.

8 Birch NC, Sly C, Brooks S, et al. Antiinflammatory drug therapy after arthroscopy of the knee. A prospective, randomised, controlled trial of diclofenac or physiotherapy. J Bone Joint Surg [Br] 1993;75:650-2.

9 Moffet $\mathrm{H}$, Richards CL, Malouin F, et al. Early and intensive physiotherapy accelerates recovery postarthroscopic meniscectomy: results of a randomized controlled study. Arch Phys Med Rehabil 1994:75:415-26.

10 Ross MJ, Berger RS. Effects of stress inoculation training on athletes' postsurgical pain and rehabilitation after orthopedic injury. J Consult Clin Psychol 1996;64:406-10.

11 Vervest AM, Maurer CA, Schambergen TG, et al. Effectiveness of physiotherapy after meniscectomy. Knee Surg Sports Traumato Arthrosc 1999;7:360-4.

12 Goodyear-Smith F, Arroll B. Rehabilitation after arthroscopic meniscectomy: a critical review of the clinical trials. Int Orthop 2001;24:350-3.

13 Goodwin PC, Morrissey MC. Physical therapy after arthroscopic partial meniscectomy: is it effective? Exerc Sport Sci Rev 2003:31:85-90.

14 Thomson LC, Handoll HHG, Cunningham A, et al. Physiotherapist-led programmes and interventions for rehabilitation of anterior cruciate ligament, medial collateral ligament and meniscal injuries of the knee in adults (Cochrane Review). Cochrane Library. Issue 4. Chichester: John Wiley \& Sons, Ltd, 2004. 


\section{Maritime health care}

\section{J Putnam}

\section{The delivery of health care to seafarers is described}

M aritime medicine refers to providing medical care for seafarers ${ }^{1}$ including the people working in the merchant marine, the commercial fishing fleets, offshore oil platforms, and the cruise ship industry, the segment best known to most of us. It also includes provision of care through shore based pre-employment and seafarer consultation clinics. These various maritime medical practices present very different alternatives to the clinics, emergency rooms, and hospital environments where most of us practice. They may provide a working vacation (often emphasis on "work"), a few months sabbatical, or a career.

Practicing medicine at sea incorporates many aspects of "wilderness medicine." Dr Warren Bowman's editorial in Wilderness Medicine, "Perspectives on being a wilderness physician" describes wilderness medicine as medical care delivery in situations where "definitive medical care may be hours or days away because of distance, adverse environmental conditions, lack of transportation, or difficulties in communication." This definition applies to work in parks, forests, and other designated wilderness areas, but is equally applicable in other environments including Antarctic research stations, remote clinics in developed countries, healthcare facilities in many "third world" countries, rural or urban locales after natural disasters, and in most of the cruise industry.

Cruise medicine provides the challenges and rewards of practicing medicine in a resource limited environment while providing the opportunity to travel to interesting, sometimes very remote, places and to meet people also intrigued by international travel and ecotourism. Numerous opportunities are available. Large ships cruise the more typical "tourist routes" of Europe, the Caribbean, and Alaska and have clinics that may be similar to a medical office at home. Smaller expedition vessels are more likely to travel to geographically remote areas, usually with specific educational themes, but you may find your cabin is also your minimally equipped office.

\section{"Over enough time, anything and everything will happen."}

This is a work environment very different from home, where wilderness medicine training, skills, and experience are invaluable. The optimal doctor for work on a cruise vessel is a family doctor or general internist who initially spent a couple of years in a surgical residency and ended up in accident and emergency. Unfortunately for the industry, that describes very few of us. The work includes that of a typical family practice and emergency room for a community of 100-5000 people, depending on the size of the vessel. Over enough time, anything and everything will happen. Patients are seen regularly for acute illnesses and exacerbations of chronic disease, acute trauma care (passenger sports/recreation injuries and crew occupational injuries), behavioural health for both passengers and crew, and ongoing management of chronic diseases for the crew, who may be on board for 10 months and are often not fluent in English. In addition, the doctor will have to deal with any medical emergencies and may be simultaneously supervising control of an influenza or Norovirus epidemic. Unlike at home, the doctor will be expected to provide the "999/911" emergency services, see patients in the outpatient clinic, accident and emergency, and infirmary (all of which are usually the same place), while often also serving as the laboratory and radiology technician, pharmacist, mental health consultant, physiotherapist, epidemiologist, visiting nurse service, clerk, and billing agent. Most companies hire primary care or accident and emergency trained doctors and nurses trained in accident and emergency or intensive care, but there are positions available for other specialists with extensive generalist experience outside of their specialty. As many of these vessels have only one doctor, there are a very limited number of positions available for physician assistants and nurse practitioners, both usually filling nursing positions.

Although you read about the newest vessels in the industry, many active vessels were built over the last $30+$ years, so shipboard medical facilities are quite variable. They may be fairly extensive on large, newer vessels, where your main limitations are manpower, remoteness, and the inability to do sophisticated testing. On smaller expedition vessels, the doctor may be the entire medical staff, with limited basic testing and treatment options available. The expedition vessels are also less likely to have available shore side resources than vessels travelling on the tourist routes.

Making a diagnosis and treatment plan for a patient's problem in an environment of sometimes very limited resources is the most unique challenge for the shipboard doctor. Those limitations can be magnified when patients neither recognise nor accept that they are in a wilderness environment. Passengers may be unprepared for the rigors of travel and poorly acclimatised to the voyage environment. They usually eat more high calorie/high salt food and may not have their medicines because they have forgotten them or their luggage was lost. Advertising and the media have glamorised the cruise industry, leading many passengers to expect the same level of care on board a cruise ship that they might find in a large urban hospital. Although a large cruise ship may in fact be better equipped than the local hospital in some ports of call, diagnostic equipment and therapeutic options remain limited. On an expedition ship in many parts of the world, such as the Antarctic peninsula, there are no shore side services, and the doctor may be the only resource available for days. Evacuating a patient from a ship is difficult at best; in many places in the world, it is impossible.

The challenges may be great but so are the rewards. Should you find this a field of medicine that might appeal to you, some resources are listed that will help you learn more about medical practice in the cruise industry.

\section{RESOURCES}

- American College of Emergency Physicians Section of Cruise Ship \& Maritime Medicine; PO Box 619911, Dallas, TX 75261, USA. The section publishes an approximately quarterly newsletter that includes a list of many of the cruise companies and the contact person for their medical department. This can also be accessed from the ACEP website. www.acep.org.

- American College of Emergency Physicians Health Care Guidelines on Cruise Ship Medical Facilities, Approved by the ACEP Section of Maritime and Cruise Ship Medicine. 
Oct 2000. Available from ACEP or from its website in the section of Cruise Ship and Maritime Medicine.

- Harrison T, ed. Cruise medicine. 2nd ed. Annapolis, MD: Maritime Health Systems, Ltd, 1999. Available from the ACEP bookstore.

- International Council of Cruise Lines, 2111 Wilson Boulevard, 8th Floor, Arlington, VA 22201. An independent council that promotes all aspects of the cruise industry, with membership by the larger companies in the cruise industry.
- Web sites of the individual cruise companies, including large companies and small ecotourism companies that may not be listed by either of the above groups. ICCL only represents its members, and the ACEP Cruise Medicine section only involves those companies whose medical advisors choose to participate.

\section{Br J Sports Med 2005;39:693-694. doi: $10.1136 /$ bjsm.2004.016964}

Correspondence to: Dr Putnam, Maridocs, Des Moines, WA 98198, USA; idpjkg@earthlink. net
Competing interests: I have worked for or consulted for Semester at Sea/Institute for Shipboard Education, World Explorer Cruises, Cruise West, and Lindblad Expeditions. I have presented at Wilderness Medical Society and SAILS conferences on the subject of Maritime Health.

\section{REFERENCES}

1 ILO Convention 164 (1987). Concerning health protection and medical care for seafarers. Geneva, 8 October, 1987.

2 Bowman WD. Perspectives on being a wilderness physician: is wilderness medicine more than a special body of knowledge? Wilderness Environ Med $2001 ; 12: 165-7$

\section{$\mathrm{ECHO}$}

\section{Marathons confuse diagnosis of $\mathrm{MI}$}

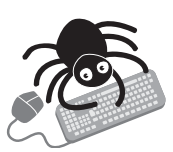

Please visit the British Journal of Sports Medicine website [www. bjsportmed. com] for a link to the full text of this article. small study of non-elite marathon runners has cast new light on cardiac troponins,
with implications for diagnosing non-ST elevation myocardial infarction (MI).
International recommendations of raised troponin T value as an indicator of acute MI
are not definitive, it seems, if the subject has engaged in prolonged exercise.

Cardiac troponin $\mathrm{T}$ ( $\mathrm{cTnT}$ ) values in blood rose to $>0.01 \mu \mathrm{g} / \mathrm{l}$ from below $0.01 \mu \mathrm{g} / \mathrm{l}$ - the detection limit of the assay-among more than three quarters of 72 non-elite runners in the London marathon. In almost $60 \%$ of them values were $>0.03 \mu \mathrm{g} / \mathrm{l}$; in $36 \%>0.05 \mu \mathrm{g} / \mathrm{l}$ - the current recommended cut off for acute MI-and in $11 \%>0.1 \mu \mathrm{g} / \mathrm{ml}$. Ischaemia was not evident on electrocardiography (ECG), but after the race the ratio of early to late left ventricular filling (E:A) dropped significantly. Raised cTnT was unaffected by age, race time, or changes in ventricular filling. Such results, when combined with dyspnoea, chest tightness, and abnormal ECG findings sometimes seen in highly trained athletes, may confuse the picture further, so caution is advised.

Data came from 2002/3 London marathons, from reputedly healthy runners with no cardiovascular disease. Each provided samples for cTnT assay and had 12 lead ECG and echocardiography 24 hours before the race and within 30 minutes after finishing.

Cardiac troponins have become linchpins of diagnosis of acute MI. Much less is known about their behaviour in the ever growing numbers competing in city marathons than in highly trained endurance athletes, and this merits further investigation, along with long term effects and changed diastolic function.

\ Shave RE, et al. Heart 2005;91:12-20. 